\title{
Establishment of immortalized murine mesothelial cells and a novel mesothelioma cell line
}

\author{
Walter Blum $^{1} \cdot$ László Pecze $^{1} \cdot$ Emanuela Felley-Bosco ${ }^{2}$. \\ Janine Worthmüller-Rodriguez ${ }^{1}$ Licun $\mathrm{Wu}^{3} \cdot$ Bart Vrugt $^{4}$ • Marc de Perrot ${ }^{3}$. \\ Beat Schwaller ${ }^{1}$
}

Received: 22 December 2014 / Accepted: 2 March 2015 / Published online: 15 April 2015 / Editor: T. Okamoto

(C) The Author(s) 2015. This article is published with open access at Springerlink.com

\begin{abstract}
Mesothelial cells are susceptible to asbestos fiber-induced cytotoxicity and on longer time scales to transformation; the resulting mesothelioma is a highly aggressive neoplasm that is considered as incurable at the present time Zucali et al. (Cancer Treatment Reviews 37:543-558, 2011). Only few murine cell culture models of immortalized mesothelial cells and mesothelioma cell lines exist to date. We generated SV40immortalized cell lines derived from wild-type (WT) and neurofibromatosis 2 (merlin) heterozygote ( $\mathrm{Nf2}+/-$ ) mice, both on a commonly used genetic background, C57Bl/6J. All immortalized mesothelial clones consistently grow in DMEM supplemented with fetal bovine serum. Cells can be passaged for more than 40 times without any signs of morphological changes or a decrease in proliferation rate. The tumor suppressor gene $N F 2$ is one of the most frequently mutated genes in human mesothelioma, but its detailed function is still un-
\end{abstract}

Beat Schwaller

Beat.Schwaller@unifr.ch

1 Department of Medicine, University of Fribourg, Route AlbertGockel 1, 1700 Fribourg, Switzerland

2 Laboratory of Molecular Oncology, Clinic of Oncology, University Hospital Zürich, Haeldeliweg 4, 8044 Zurich, Switzerland

3 Thoracic Surgery Research Laboratories, Division of Thoracic Surgery, Toronto General Hospital, University Health Network, University of Toronto, Toronto, ON, Canada

4 Institute of Surgical Pathology, University Hospital Zürich, Zürich, Switzerland known. Thus, these genotypically distinct cell lines likely relevant for malignant mesothelioma formation are expected to serve as useful in vitro models, in particular to compare with in vivo studies in mice of the same genotype. Furthermore, we generated a novel murine mesothelioma cell line RN5 originating from an Nf2+/mouse subjected to repeated crocidolite exposure. RN5 cells are highly tumorigenic.

Keywords Mesothelioma $\cdot$ Asbestos $\cdot$ Mouse cell lines $\cdot$ Cell models $\cdot$ Immortalized $\cdot$ SV40

\section{Introduction}

Malignant mesothelioma (MM) is an aggressive form of cancer with an average survival time of about $1 \mathrm{yr}$; up to date, conventional therapies have proven to be rather unsuccessful (Rascoe et al. 2012). Tumors are derived from transformed mesothelial cells, a cell type that is covering the internal cavities of the pericardium, peritoneum, and the pleura. The mesothelium is a cell monolayer consisting of flat cells, forming the barrier between the tunica mucosae parietalis and visceralis. Pleural MM is linked in up to $80 \%$ of the cases with asbestos exposure (Carbone et al. 2011). Besides exposure to asbestos fibers leading to MM formation, other etiologies including genetic disposition and, rarely, ionizing radiation were found associated to MM (Carbone et al. 2011; Chirieac et al. 2013). In addition, SV40 oncoproteins [large $\mathrm{T}$ and small $\mathrm{t}$ antigen (TAg and tag, respectively)] together with asbestos fibers were demonstrated to be co-carcinogenic (Bocchetta et al. 2000; Kroczynska et al. 2006). Animal models to study MM 
were long hampered due to the slow time-course between asbestos exposure and MM formation, a period that ranges from 20 to $40 \mathrm{yr}$ in humans (Bianchi et al. 1997). Exposure of wild-type (WT) mice to asbestos fibers, even at high doses, results in the formation of MM only in a small fraction of animals $(<33 \%)$ (Altomare 2005). Mutations in the NF2 gene have been found in about $40 \%$ of human mesothelioma (Bianchi et al. 1995; Sekido et al. 1995; Deguen et al. 1998), and the influence of $\mathrm{Nf} 2$ heterozygosity has been investigated in mice; $\mathrm{Nf} 2$ heterozygosity was shown to result in $\mathrm{MM}$ at high frequency $(85 \%)$ within $1 \mathrm{yr}$ after repetitive asbestos exposure (Altomare 2005). In another transgenic mouse model, SV40 TAg expression driven by the mesothelin promoter leads to asbestos-induced $\mathrm{MM}$ in $<1$ yr (Robinson et al. 2006). However, for many aspects of MM research, in particular for the investigation of the early steps of mesotheliomagenesis, cell lines are of utmost importance, providing relatively inexpensive model systems with high throughput capacities and reducing the need for animal experiments. For the investigation of the early steps of MM formation, immortalized cell lines derived from primary mesothelial cells are the tools of choice. The most used immortalized human mesothelial cell lines are MeT5A immortalized with SV40 (Ke et al. 1989) and LP9 cells immortalized with TERT1, the catalytic component of telomerase (Connell and Rheinwald 1983). Immortalized mesothelioma and mesothelial cells of mouse origin include cells isolated from the ascites of asbestosexposed wild-type $\mathrm{C} 57 \mathrm{Bl} / 6 \mathrm{~J}$ or BALB/c mice $[40$, 40L, AE17, AK7 (Cordier Kellerman et al. 2003), and AB12 (Davis et al. 1992)], SV40 TAg transgenic mice [MexTAg, line names TGM299h, TGM304i, TGM270i, and TGM266i (Robinson et al. 2006), conditional Nf2-/- mice (Jongsma et al. 2008)], and spontaneously immortalized cells obtained after prolonged culturing of primary mouse mesothelial cells (Sherwood et al. 2008). Here, we established SV40-immortalized mesothelial cells of WT mice and immortalized cells derived from (Nf2+/-) mice; all lines stem from mesothelial cells isolated from mice with $\mathrm{C} 57 \mathrm{Bl} / 6 \mathrm{~J}$ background. We expect the latter cell lines to serve as valuable models to investigate in further detail the role of $\mathrm{Nf2}$ (merlin) in vitro and to complement in vivo studies carried out in $\mathrm{Nf} 2+/-$ mice. Immortalized mesothelial cell lines were not tumorigenic, but showed anchorage-independent growth in the spheroid formation assay, thus allowing to use them as 3D cell culture models (Smalley et al. 2006). Since the role of SV40 in $\mathrm{MM}$ is still controversially discussed in the mesothelioma field (Carbone et al. 2003), the different immortalized mesothelial cell lines also provide a model to investigate the effect of inactivating p53 in different genotypes (WT, Nf2+/-). Finally, a novel murine mouse mesothelioma cell line RN5 was generated from a tumor that had developed in a crocidolite-exposed Nf2+/mouse; this line shows high tumorigenicity in vivo and persistent growth in vitro.

\section{Materials and Methods}

Isolation of mouse primary mesothelial cells and mesothelioma cells from a NF2+/- mouse. Mesothelial cells were isolated from 4 to 6 months old WT and Nf2+/- mice, all on a C57Bl/6J background using a previously described protocol (Bot et al. 2003). Briefly, mice were killed and the peritoneal cavities were exposed by incision and removal of the fur. The peritoneal cavities were washed with injecting approximately $50 \mathrm{ml}$ of phosphate-buffered saline (PBS) (Sigma, St. Louis) via a syringe equipped with a $25 \mathrm{G} \times 5 / 8^{\prime \prime}$ needle $(\mathrm{BD}$ microlance 3, Becton Dickinson AG, Allschwil, Switzerland) using a peristaltic pump and a second needle $(23 \mathrm{G}, 0.6 \times 25 \mathrm{~mm})$ to allow exit of the PBS solution. Perfusion was maintained until the exiting PBS solution was clear, i.e., devoid of mobile and poorly attached cells. Residual PBS was aspired with a syringe, and the peritoneal cavity was filled with $5 \mathrm{ml}$ of $0.25 \%$ Trypsin/EDTA solution (Gibco, Basel, Switzerland). The body temperature of mouse corpses was maintained at around $37^{\circ} \mathrm{C}$ for $5 \mathrm{~min}$ via an infrared heating lamp. The suspension containing the detached cells was collected with a syringe; cells were centrifuged for $10 \mathrm{~min}$ at $300 \times \mathrm{g}$. Cells mostly comprising primary mesothelial cells were grown in modified Connell's medium composed of Dulbecco's modified Eagle's medium (DMEM)/F12+GlutaMax (Gibco), 15\% fetal calf serum (FCS), $0.4 \mu \mathrm{g} / \mathrm{ml}$ hydrocortisone, $10 \mathrm{ng} / \mathrm{ml}$ epidermal growth factor, 1\% ITS (insulin, transferrin, selenium), $1 \mathrm{mM}$ sodium pyruvate, $0.1 \mathrm{mM}$ beta-mercaptoethanol, $1 \%$ non-essential amino acids, $1 \%$ penicillin-streptomycin, and 2\% Mycokill (PAA) (Connell and Rheinwald 1983). The Nf2 alleles (WT or mutated) were genotyped using the common forward primer (NF2_FW 5'-GGGGCTTCGGGAAACCTG G-3'), and either NF2_RV WT (5'-GTCTGGGAAGTC TGTGGAGG-3) or NF2_RV mutant (5'-CTATCAGG ACATAGCGTTGG-3') primers. The cell line RN5 was isolated from an $\mathrm{Nf} 2+/-$ mouse that was repeatedly injected with crocidolite starting at 8 wk of age $(7 \times$ $400 \mu \mathrm{g})$. Briefly, a clearly discernible tumor localized on the liver was dissected from the mouse $21 \mathrm{wk}$ after the first injection. The tissue was incubated in a $0.25 \%$ Trypsin/EDTA solution for $10 \mathrm{~min}$; tumor cells were dissociated by mild trituration and cultured in DMEM, $10 \%$ fetal bovine serum (FBS, Gibco, Basel, Switzerland), and $1 \%$ PS $(100 \mathrm{U} / \mathrm{mL}$ penicillin and $100 \mu \mathrm{g} / \mathrm{mL}$ streptomycin). 
Cell culture and growth curves. Primary mesothelial cells from the two genotypes (WT and Nf2+/-) were immortalized by transduction with SV40 small and large T antigen. Transduction was performed using lentivirus that were produced as previously described (Blum and Schwaller 2013). The transfer plasmid $\mathrm{pCMV} / \mathrm{TO}$ SV40 was obtained from Addgene (\# 22298). Primary cells were seeded at a density of 10,000 cells $/ \mathrm{cm}^{2} ; 24 \mathrm{~h}$ post-seeding cells were transfected with lentivirus at an MOI of 5. Proliferation was monitored using the Incucyte Live Cell Imaging System (Essen Bioscience, Michigan). After passaging, cells were seeded at a very low density $\left(1-2\right.$ cells $\left./ \mathrm{cm}^{2}\right)$ in $10-\mathrm{cm}$ Petri dishes. Individual and well-delimited clones were picked and transferred into 96-well plates and frozen at passages 710 following standard protocols. 3-(4,5-Dimethylthiazol2yl)-2,5-diphenyltetrazolium bromide (MTT) assay was performed as previously described (Blum and Schwaller 2013).

Western blot assay for mesothelin, SV40 large T-antigen, and $\beta$-actin. Cells were grown in $75-\mathrm{cm}^{2}$ cell culture flasks (TPP, Trasadingen, Switzerland) until they reached nearly $100 \%$ confluence. Subsequently, cells were trypsinized and pelleted $(500 \times g, 3 \mathrm{~min})$, then washed three times with CMFPBS. Proteins were extracted with RIPA buffer [50 mM Tris, $150 \mathrm{mM} \mathrm{NaCl}, 0.1 \%$ sodium dodecyl sulfate (SDS), $0.5 \%$ sodium deoxycholate, $1 \%$ Triton X-100, pH 7.4], incubated for $30 \mathrm{~min}$ on ice, and centrifuged $30 \mathrm{~min}$ at $17,000 \times \mathrm{g}$ at $4{ }^{\circ} \mathrm{C}$, and the supernatant was collected. The DC assay (BioRad) was performed to quantify the proteins following the manufacturer's protocol. Protein samples were separated on a $10 \%$ polyacrylamide SDS gel and transferred onto nitrocellulose membranes. Membranes were checked with Ponceau S staining for equal loading. Membranes were blocked with 5\% milk PBS for $1 \mathrm{~h}$ at room temperature and incubated overnight at $4^{\circ} \mathrm{C}$ with antibodies recognizing mesothelin (dilution 1:2,000; Santa Cruz Biotechnology, Santa Cruz, CA, clone B-3), SV40 large T-antigen (1:2000; Santa Cruz Biotechnology, Pab101), and $\beta$-actin $(1: 20,000$; Sigma-Aldrich, clone ac-74).
Secondary biotinylated antibodies were used at a dilution of 1:20,000, and the ABC system (Vectastain, Vector Laboratories, Burlingame, CA) was applied. The HRP substrate (Millipore, Luminata Forte) was incubated for $3 \mathrm{~min}$ on the membrane and analyzed on a Western blot reader (FluorChem E System, Bucher Biotec, Basel, Switzerland).

Soft agar assay. The procedure of the soft agar assay was adapted from a previously described protocol (Provost et al. 2012). Shortly, $0.5 \%$ of base agar was prepared by melting $1 \%$ of agar (LuBioScience, Lucerne, Switzerland) in a microwave oven and adding of prewarmed $\left(40^{\circ} \mathrm{C}\right) 2 \times$ RPMI completed with 20\% FCS and antibiotics (penicillin-streptomycin) and allowed to equilibrate to $37^{\circ} \mathrm{C}$. Equal volumes of the solutions were mixed to give final concentrations of $0.5 \%$ agar, $1 \times$ RPMI, and $10 \%$ FCS. One milliliter was added per well in six-well plates and set aside to solidify. Top agar was prepared by melting $0.7 \%$ agarose (PeqLab, Erlangen, Germany) in a microwave oven and cooled down to $40^{\circ} \mathrm{C}$ and then supplemented with an equal volume of $2 \times$ RPMI with $20 \%$ FCS also at approximately $40^{\circ} \mathrm{C}$. Cells (5000) in a volume of $1 \mathrm{ml}$ of $0.35 \%$ agarose in $1 \times \mathrm{RPMI}+10 \% \mathrm{FCS}$ was added to the solidified agar. Plates were incubated for $17 \mathrm{~d}$ in a humidified incubator at $37^{\circ} \mathrm{C}$ with $5 \% \mathrm{CO}_{2}$. Twice per week, the plates were supplied with $0.5 \mathrm{ml}$ of cell culture medium.

Subcutaneous xenograft model. WT C57Bl/6J mice (6-8 wk old; $n=3-5$ animals depending on the group) were inoculated s.c. with $4 \times 10^{5}$ cells by injection of the cell suspension $(50 \mu \mathrm{l}$ in PBS containing 20\% matrigel) into the right dorsal flank. Cells included immortalized mesothelial cells (iMeso-WT1 and iMeso-NF3), the MM cell line RN5, and the previously described murine MM cell line AK7 (Cordier Kellerman et al. 2003), the latter also on a C57Bl/6 J background. Tumors were measured with a caliper weekly as soon as palpable; the volume was calculated using the formula $a \times b^{2} \times 0.5$, where $a$ is the large and $b$ the small diameter of an ellipse. For the
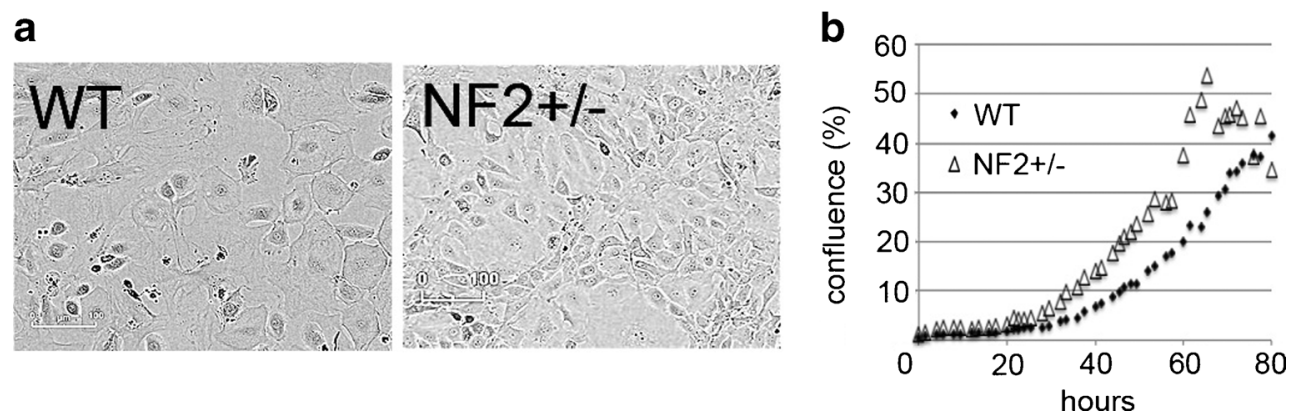

Figure 1. Morphology $(a)$ and growth characteristics $(b)$ of primary mesothelial cells isolated from WT and $\mathrm{Nf} 2+/-$ mice, both grown in Connell's medium. (a) Mesothelial cells from both genotypes show the typical "cobblestone-like" morphology. (b) Nf2+/- cells show slightly increased proliferation compared to WT cells; representative growth curves for $n>3$ experiments are shown. 
immunohistochemistry, deparaffinized sections were subjected to antigen retrieval using sodium citrate, $\mathrm{pH} 6$, then were processed as previously described (Frei et al. 2011). Primary

a
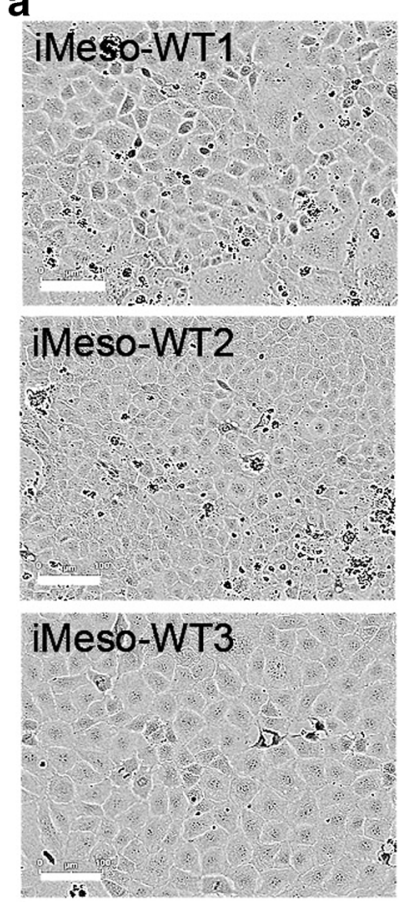

b
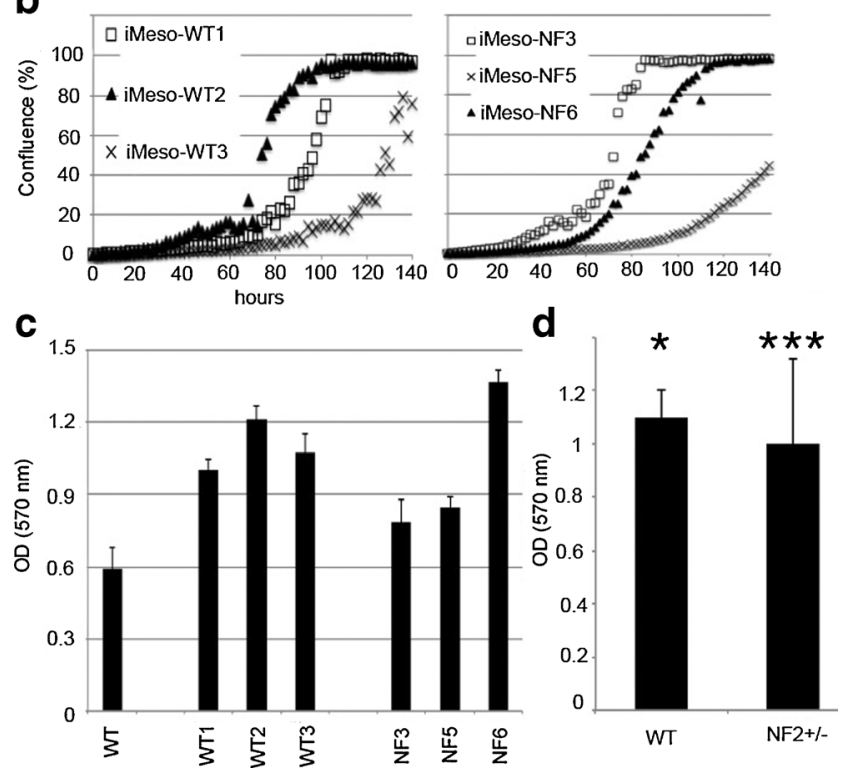

Figure 2. Immortalized mesothelial cell morphology and cell growth in DMEM $+10 \%$ FBS. (a) Brightfield images were taken with the Incucyte Live Cell Imaging system (FLR 10×) and show immortalized mesothelial cell clones derived from WT and Nf2+/- mice. The typical "cobblestonelike" morphology is conserved; all clones show a higher proliferation rate than their nonimmortalized primary counterparts (scale bar: $100 \mu \mathrm{m})$. (b) Representative Incucyte growth curves of WT and Nf2+/- clones. (c) MTT signals obtained at $144 \mathrm{~h}$ postseeding using all cells and cell lines as in Fig. 1 and $2 a$. (d) MTT data grouped for three clones each of the genotypes WT and $\mathrm{Nf} 2+/-$ in comparison to the primary mesothelial cells $\left({ }^{*} p<0.05 ; * * * p<0.0005\right)$. antibodies used were anti-wide spectrum cytokeratin and antivimentin (Abcam Ab9377, 1:100 and Ab92547, 1:500, respectively).

Immunofluorescence experiments. Cells were seeded on glass coverslips and incubated with the appropriate cell culture medium until a confluence of $70-90 \%$ was reached. Then, the cells were fixed with $4 \%$ PFA, permeabilized with $1 \%$ Triton $\mathrm{X}-100$, and blocked with TBS solution containing donkey serum $(10 \%)$. Cells were incubated overnight at $4{ }^{\circ} \mathrm{C}$ with the primary antibodies at the indicated dilutions: mouse monoclonal anti-pan-cytokeratin, clone Lu-5 (BMA Biomedicals, Augst, Switzerland, T-1302; 1:500), or rabbit polyclonal antidesmin (Sigma D8281; 1:500). After washing, the cellcontaining coverslips were incubated with secondary antibodies for $1 \mathrm{~h}$ with either DyLight488-conjugated donkey antimouse IgG (Jackson Immunoresearch Laboratories, West Grove, PA; 1:1,000) or Cy3-conjugated donkey anti-rabbit IgG (Jackson Immunoresearch Laboratories; 1:1,000). The cells were counterstained with DAPI (Molecular Probes, Eugene, OR; $5 \mu \mathrm{g} / \mathrm{ml}$ ) and mounted with Hydromount solution (National Diagnostics, Atlanta, GA). Images were acquired with a LEICA fluorescent microscope DM6000B (Wetzlar, Germany) equipped with a Hamamatsu camera C4742-95 (Bridgewater, NJ).

\section{Results}

Immortalized primary mouse mesothelial persistently grow in vitro. Primary mesothelial cells isolated from WT and $\mathrm{Nf2}+/-$ mice were initially grown in Connell's medium, a

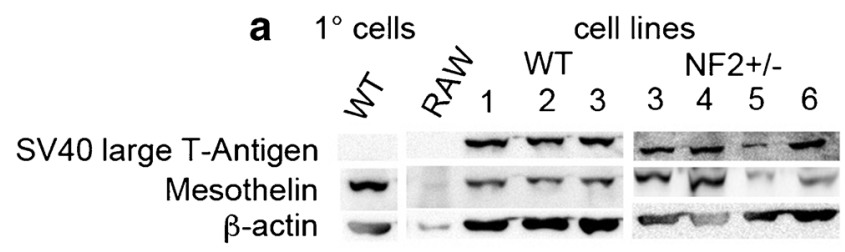

b

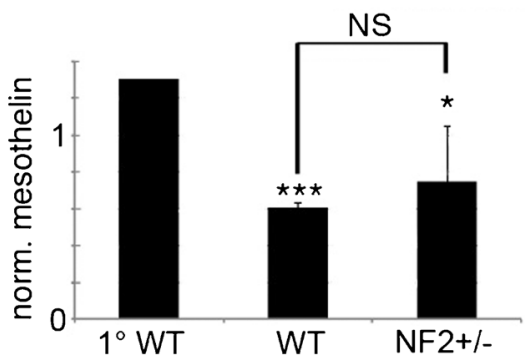

Figure 3. Western blot for SV40 large T antigen and mesothelin in WT and $\mathrm{Nf} 2+/-$ cells in comparison of the primary immortalized clones from WT and Nf2+/- mesothelial cells. (a) Western blot signals from a representative experiment. The Western blot signals for $\beta$-actin were used for the normalization of the mesothelin expression levels. (b) Quantitative analysis of a representative Western blot $(n=3$ samples per genotype, $p<0.05$ grouped primary cells vs. grouped immortalized cell lines). 


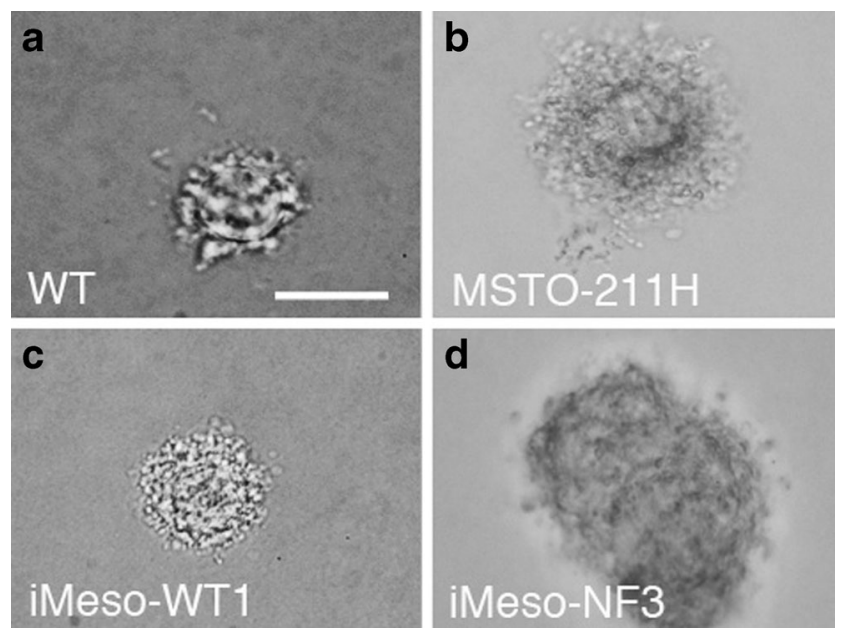

Figure 4. Colony formation assay. (a) Primary WT mesothelial cells were able to form colonies in plates with soft agar, as well as the human MM-derived cell line MSTO-211H (b) used as a positive control (derived from a biphasic mesothelioma). Selected colonies typically representing the morphological appearance of immortalized cells iMeso-WT1 (c), and iMeso-NF3 (d) are shown. Scale bar: $250 \mu \mathrm{m}$.

medium previously shown to be optimal for mesothelial cell growth (Connell and Rheinwald 1983). Growth of cells from both genotypes was faster then in the medium used for the further experiments, i.e., in DMEM supplemented with $10 \%$ FBS (data not shown). Primary mesothelial cells from WT and $\mathrm{Nf} 2+/-$ mice grown in DMEM showed the typical cobblestone-like morphology similar as, e.g., the human mesothelioma cell line ZL55 consisting of cells with an epithelioid morphology used in previous studies (Blum and Schwaller 2013) (Fig. 1a). As shown before for WT primary mouse mesothelial cells (Robinson et al. 2006), mesothelial cells from the two genotypes grew in vitro for approximately 810 passages, followed by a prolonged period of quiescence lasting for up to several weeks before finally dying.

\section{a}
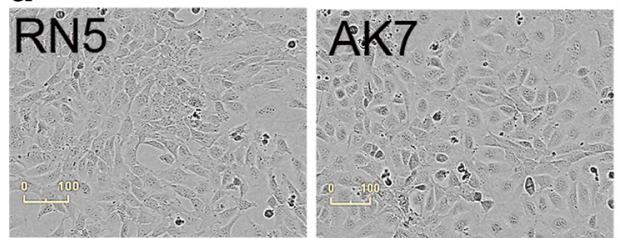

C
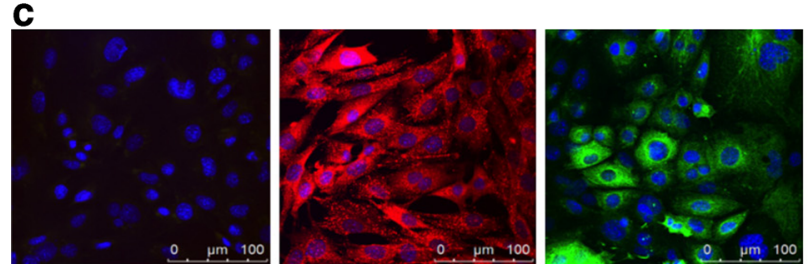

Of note, primary mesothelial cells (initial density, 1500 cells $/ \mathrm{cm}^{2}$ ) from $\mathrm{Nf} 2+/-$ mice showed slightly enhanced proliferation compared to the cell lines generated from WT mice (Fig. 1b). From the initial batch of immortalized cells, by limited dilution, clones derived from three colonies for each genotype, likely originating from a single cell, thus representing single clones, were isolated, expanded, and stored for further usage. Unlike the primary mesothelial cells, SV40-immortalized mesothelial cell clones (iMeso-WT, iMeso-Nf2) grew rapidly in DMEM supplemented with $10 \%$ FBS (Fig. 2). Cells from both genotypes showed persistent cell proliferation even under low serum conditions $(2 \%)$ and all survived for prolonged periods of time ( $>120 \mathrm{~h}$ ) under very low serum conditions $(0.5 \%)$, however without signs of proliferation evidenced by lack of mitotic cells (data not shown). All cultured mesothelial cells showed the typical cobblestonelike morphology like primary mesothelial cells from WT and Nf2+/- (Fig. 2a). All clones irrespective of genotype maintained their typical pavement-like morphology, and moreover, immortalized cell lines of both genotypes showed an increased cell proliferation rate when compared to the initial primary mesothelial cells (Fig. 2b) evidenced from real-time growth curves, as well as from the results of an independent proliferation assay, the MTT assay, carried out $144 \mathrm{~h}$ postplating (Fig. 2c). Of note within one genotype (WT and $\mathrm{Nf2+/-}$, growth rates of the various clones were considerably different, e.g., WT clones 1, 2, and 3 reached the plateau (close to $100 \%$ confluence) within 100,80 , and $\approx 140 \mathrm{~h}$, respectively (Fig. 2b); similar proliferation rates were also observed for $\mathrm{Nf} 2+/-$ clones. This was also confirmed using the MTT assay (Fig. 2c), when clones were grouped according to their genotype. No significant differences in growth rates were evident between the group of WT and NF2+/- clones (Fig. 2d). All SV40-immortalized mesothelial cell lines (clones) were cultured for more than 40 passages without showing any signs of
Figure 5. Morphology and growth of the murine mesothelioma cell lines $\mathrm{RN} 5, \mathrm{AK} 7$, and AB12 in vitro. (a) Epithelioid morphology of RN5 and AK7 compared to biphasic or mixed type morphology of AB12 cells. Cell size, morphology, and growth characteristics $(b)$ are strikingly similar for RN5 and AK7 cells. (c) Immunofluorescene images of RN5 cells show a rather homogeneous staining for desmin (red). Pancytokeratin staining (green) shows a typical mosaic pattern; cell nuclei were labeled with DAPI (blue). (d) Western blot analysis $(30 \mu \mathrm{g}$ total protein/lane) showing the expression of mesothelin in the three murine mesothelioma cell lines RN5, AK7, and AB12. 
a decreased growth and/or changes in cell morphology. A stock was produced at passage number 7-10 and frozen for long-term storage.

Characterization of primary and immortalized mesothelial cells. As expected, primary mesothelial cells of either genotype (WT, Nf2+/-), as well as the murine macrophage cell line RAW264.7 (Raschke et al. 1978), did not express TAg evidenced by Western blotting (Fig. 3a). Strong TAg expression was observed in all primary mesothelial cell clones transfected with the lentivirus coding for TAg/tag (Fig. 3). All cells (primary, immortalized) of mesothelial origin were clearly positive for mesothelin, a mesothelial cell-specific marker. The single positive band on the Western blots had a relative molecular mass $\left(M_{\mathrm{r}}\right)$ of $69 \mathrm{kDa}$ corresponding to the mesothelin precursor protein. Western blot quantification revealed decreased mesothelin levels in immortalized cell clones from both genotypes compared to primary cells WT mice (Fig. 3b). Immunohistochemical analysis showed homogeneous positive staining for desmin and mosaic-like expression for Pan-cytokeratin in all examined cell lines including the primary mesothelial cultures, the immortalized cells, and the tumor-derived RN5 cells. A typical example for RN5 cells is shown in Fig. 5c.

\section{Primary mesothelial cells and SV40-immortalized mesothelial} cells form colonies in the soft agar assay. Since immortalized mesothelial cell clones of both genotypes (WT, Nf2+/-) also proliferated in low-serum ( $2 \%$ ) conditions, a feature often observed in transformed cells, we investigated another aspect related to cell transformation, i.e., their ability to form anchorage-independent colonies in a semisolid medium. Cells from the various clones grew in soft agar, and images were taken after $17 \mathrm{~d}$ (Fig. 4). We then also tested primary mesothelial cells from WT mice; they were also able to form colonies of $\geq 250 \mu \mathrm{m}$ diameter after $17 \mathrm{~d}$ of growth in anchorage-independent conditions (Fig. 4). These findings are in line with results on human primary mesothelial cells, where colony formation in semisolid medium has been reported before (La Rocca and Rheinwald 1985). Thus, anchorageindependent growth of primary mesothelial cells appears not to be restricted to cells of human origin; moreover, the soft agar assay does not allow to distinguish primary from immortalized or even transformed (RN5) cells. As a positive control, the human MM cell line MSTO- $211 \mathrm{H}$ was used. The sole purpose of these experiments was to test whether or not murine mesothelial cells (primary, immortalized) have the capability of growing in soft agar in an anchorage-independent way. No attempt was made to correlate the mesothelial cell's nature (primary vs. immortalized vs. transformed, from WT vs. transgenic mice) with the size of the colonies.
Morphological and growth characteristics of the novel RN5 cell line compared to $A K 7$ and AB12 mouse mesothelioma cell lines. We isolated a primary tumor obtained after repeated injection of asbestos fibers in the peritoneum of an $\mathrm{Nf2}+/-$ mouse. At the age of $29 \mathrm{wk}$, many tumors covered the spleen and the liver of this mouse. From a well-delimited piece of tumor tissue, the tumor cells were isolated by trypsinization of the tumor tissue followed by plating in DMEM. These cells, named RN5, have a similar morphology like AK7 cells in vitro, i.e., both cell lines show an epithelioid morphology, nearly as cobblestone-like as primary mesothelial cells (compare Fig. 5a and Fig. 1a). AB12 cells show sarcomatoid morphology in most cells and some epithelioid cells hinting towards a biphasic type. All three cell lines show fast

a

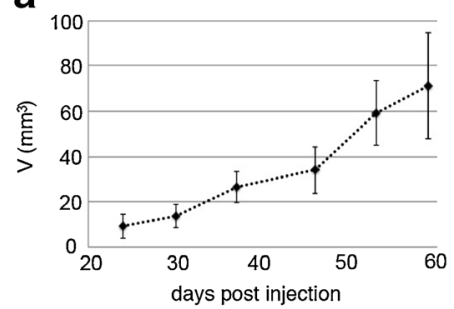

b
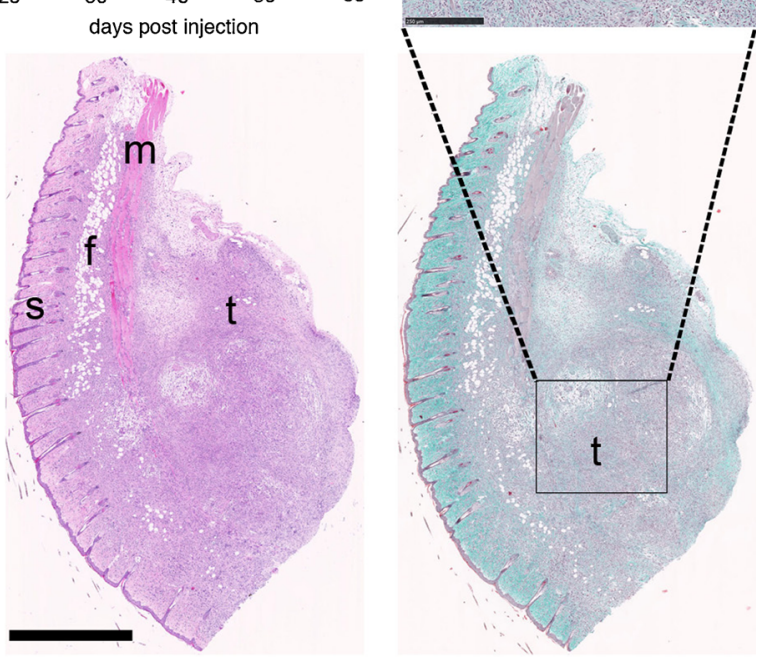

d

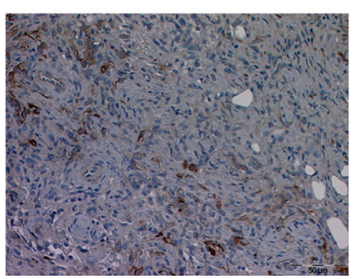

e
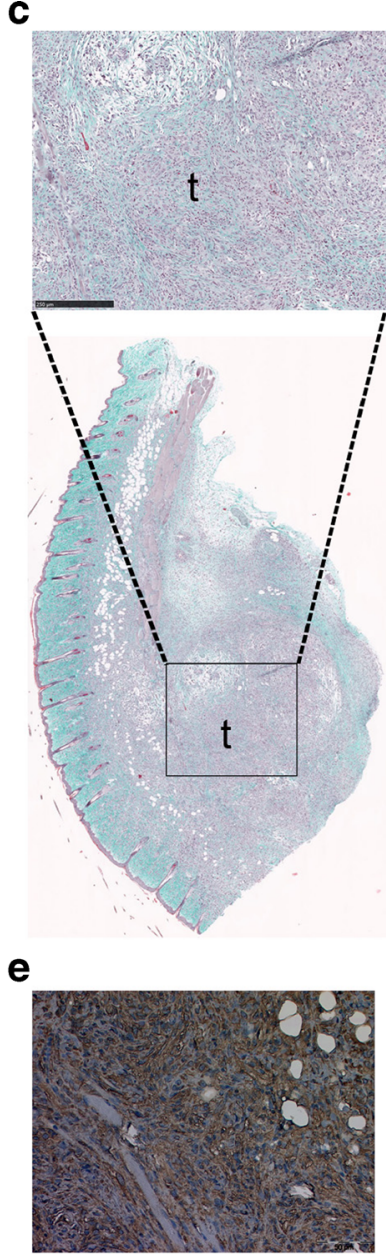

Figure 6. RN5 subcutaneous xenograft. (a) Xenograft growth monitored weekly $24 \mathrm{~d}$ after inoculation ( $n=4$ mice). (b) Histological sections of the xenograft stained by hematoxylin and eosin (left) and Goldner (right) showing the skin $(s)$, the subcutaneous fat tissue $(f)$, and muscle $(m)$. Scale bar: $1 \mathrm{~mm}$. (c) Magnification emphasizing tumor tissue $(t)$ from the Goldner colored section. Scale bar: $250 \mu \mathrm{m}$. Immunohistochemical analysis showed expression of vimentin $(d)$ with strong coexpression of pan-cytokeratin $(e)$ in part of the tumor cell population. 
proliferation and similar growth characteristics as evidenced by real-time imaging experiments (Fig. 5b).

RN5 cells form tumors in the subcutaneous xenograft model. Primary mesothelial cells from WT mice, immortalized cells iMeso-WT1, iMeso-NF3, and mesothelioma cells from the lines AK7 and RN5, all derived from a C57Bl/6J background, were injected $\left(4 \times 10^{5}\right.$ cells $)$ subcutaneously into the right flank of $\mathrm{C} 57 \mathrm{Bl} / 6 \mathrm{~J}$ mice. Tumor formation was observed in all four mice injected with RN5 cells (Fig. 6a). No macroscopically evident (palpable) tumors were observed in mice injected with primary mesothelial cells of WT origin or with immortalized cells (iMeso). No tumors developed after injection of the selected number of AK7 cells; a significantly higher number $\left(>2 \times 10^{6}\right.$ cells) has been reported to be required in order to induce macroscopic tumors in this experimental model (Cordier Kellerman et al. 2003). The tumor tissue originating from RN5 cells isolated $59 \mathrm{~d}$ after injection was white in color and rather solid at palpation. On histological sections subjected to a Goldner staining, a fibrous stroma was evident (Fig. 6c). Histological examination of tumors (Fig. 6b) derived from RN5 cells isolated $59 \mathrm{~d}$ after injection revealed a proliferation of atypical cells with a sarcomatoid morphology. The tumor was mainly confined to the subcutaneous tissue with infiltration of small clusters as well as individual cells into the dermis. Immunohistochemically, the tumor cells were positive for vimentin with strong coexpression of pancytokeratin in part of the cell population (Fig. 6d, e). No expression was seen for WT1 and calretinin (data not shown). The morphology combined with staining for vimentin and pancytokeratin is consistent with a sarcomatoid mesothelioma.

\section{Discussion}

Cell culture systems in vitro represent helpful models to investigate tumor formation and, together with murine cancer models in vivo, have become a fundamental tool in Life Sciences (Balmain 2002). We generated simultaneously and on the same C57Bl/6J genetic background SV40immortalized murine peritoneal mesothelial cell clones from two different genotypes: WT and Nf2+/-. This strategy offers several advantages in studies aimed to characterize, e.g., nanomaterial toxicity (asbestos, novel nanomaterials). The most obvious is that it allows characterizing the influence of $\mathrm{Nf} 2$ (merlin) without concerns about the influence of a genetic drift observed in inbred strains with mixed background or in C57BL/6 substrains (Zurita et al. 2011). Our murine cell lines derived from $N f 2$ heterozygous mice provide a model system to investigate $\mathrm{Nf} 2$ (merlin) function and to possibly investigate the mechanisms leading to the inactivation of the nonmutated allele. Indeed, although Nf2-deficient murine cell lines are available (Jongsma et al. 2008), they are, in addition, also deficient for cyclin-dependent kinase inhibitor 2A $(C d k n 2 a)$ and, moreover, are on a mixed genetic background.

Mesothelial lines immortalized with SV40 T antigens have allowed highlighting the importance of $\mathrm{p} 53$ in maintaining genomic stability (Levresse et al. 2000; Pietruska and Kane 2007). We confirmed that SV40 T antigen expression, although accelerating the rate of the cell cycle, consistent with previous data (reviewed in An et al. 2012), is not sufficient to transform mesothelial cells (Cleaver et al. 2014). Therefore, they may constitute a suitable model to investigate early steps of mesothelial transformation, however also taking into consideration the limitations of such a model.

The establishment of the novel mouse mesothelioma cell line RN5 originated from a heterozygote $\mathrm{Nf} 2+/-$ mouse on a $\mathrm{C} 57 \mathrm{Bl} / 6 \mathrm{~J}$ background is expected to be useful also for in vivo investigations on (1) the modulation of tumor growth by decreased merlin levels (possibly linked to loss of heterozygosity), (2) the role of the immune system in asbestos-mediated mesothelioma development, and (3) the role of other stromal components in tumorigenesis. Tumorigenicity could be investigated in WT vs. the large variety of $\mathrm{C} 57 \mathrm{Bl} / 6 \mathrm{~J}$ derived-mice deficient in stromal components. Moreover, RN5 is the first cell line from $\mathrm{C} 57 \mathrm{Bl} / 6 \mathrm{~J}$ mice that is uniquely heterozygous for $\mathrm{Nf} 2$.

In conclusion, we have established new immortalized mouse mesothelial cell lines that provide model systems to study, e.g., mechanisms implicated in mesothelial transformation or to test for nanomaterial toxicity. We expect that these in vitro models will also help to reduce animal experimentation. The cell line RN5 was demonstrated to be fast and persistently growing in vitro and to be highly tumorigenic in syngeneic $\mathrm{C} 57 \mathrm{Bl} / 6 \mathrm{~J}$ mice. These tumor cell-exposed mice are expected to retain a functional immune response. We foresee that this in vivo model will allow for testing putative therapeutic options against malignant mesothelioma.

Acknowledgments The authors wish to thank Valérie Salicio, Simone Eichenberger, and Marlène Sanchez for excellent technical assistance and Dr. E. Campeau for providing the plasmid pCMV/TO SV40 (Addgene plasmid \#22298). The projected was supported by the Swiss National Science Foundation (SNF grant no. 130680 to B.S, SNF Sinergia grant no. 147697 to E.F.-B., M.d.P. and B.S., and the San Salvatore Foundation.

Open Access This article is distributed under the terms of the Creative Commons Attribution License which permits any use, distribution, and reproduction in any medium, provided the original author(s) and the source are credited.

\section{References}

Altomare DA (2005) A mouse model recapitulating molecular features of human mesothelioma. Cancer Res 65:8090-8095. doi:10.1158/ 0008-5472.CAN-05-2312

An P, Sáenz Robles MT, Pipas JM (2012) Large T antigens of polyomaviruses: amazing molecular machines. Annu Rev 
Microbiol 66:213-236. doi:10.1146/annurev-micro-092611150154

Balmain A (2002) Cancer as a complex genetic trait: tumor susceptibility in humans and mouse models. Cell 108:145-152. doi:10.1016/ S0092-8674(02)00622-0

Bianchi AB, Mitsunaga SI, Cheng JQ et al (1995) High frequency of inactivating mutations in the neurofibromatosis type 2 gene (NF2) in primary malignant mesotheliomas. Proc Natl Acad Sci U S A 92: 10854-10858

Bianchi C, Giarelli L, Grandi G et al (1997) Latency periods in asbestosrelated mesothelioma of the pleura. Eur J Cancer Prev 6:162-166

Blum W, Schwaller B (2013) Calretinin is essential for mesothelioma cell growth/survival in vitro: a potential new target for malignant mesothelioma therapy? Int J Cancer 133:2077-2088. doi:10.1002/ijc.28218

Bocchetta M, Di Resta I, Powers A et al (2000) Human mesothelial cells are unusually susceptible to simian virus 40-mediated transformation and asbestos cocarcinogenicity. Proc Natl Acad Sci U S A 97: 10214-10219

Bot J, Whitaker D, Vivian J et al (2003) Culturing mouse peritoneal mesothelial cells. Pathol Res Pract 199:341-344. doi:10.1078/ 0344-0338-00427

Carbone M, Pass HI, Miele L, Bocchetta M (2003) New developments about the association of SV40 with human mesothelioma. Oncogene 22:5173-5180. doi:10.1038/sj.onc. 1206552

Carbone M, Ly BH, Dodson RF et al (2011) Malignant mesothelioma: facts, myths, and hypotheses. J Cell Physiol 227:44-58. doi:10. $1002 /$ jcp. 22724

Chirieac LR, Barletta JA, Yeap BY et al (2013) Clinicopathologic characteristics of malignant mesotheliomas arising in patients with a history of radiation for Hodgkin and non-Hodgkin lymphoma. J Clin Oncol 31:4544-4549. doi:10.1200/JCO.2013.49.9616

Cleaver AL, Bhamidipaty K, Wylie B et al (2014) Long-term exposure of mesothelial cells to SV40 and asbestos leads to malignant transformation and chemotherapy resistance. Carcinogenesis 35:407-414. doi:10.1093/carcin/bgt322

Connell ND, Rheinwald JG (1983) Regulation of the cytoskeleton in mesothelial cells: reversible loss of keratin and increase in vimentin during rapid growth in culture. Cell 34:245-253

Cordier Kellerman L, Valeyrie L, Fernandez N et al (2003) Regression of AK7 malignant mesothelioma established in immunocompetent mice following intratumoral gene transfer of interferon gamma. Cancer Gene Ther 10:481-490. doi:10.1038/sj.cgt.7700594

Davis JM, Manning LS, Whitaker D et al (1992) Establishment of a murine model of malignant mesothelioma. Int J Cancer 52:881886. doi:10.1002/ijc.2910520609

Deguen BB, Goutebroze LL, Giovannini MM et al (1998) Heterogeneity of mesothelioma cell lines as defined by altered genomic structure and expression of the NF2 gene. Int J Cancer 77:554-560. doi:10. 1002/(SICI)1097-0215(19980812)77:4<554::AID-IJC14>3.0. $\mathrm{CO} ; 2-6$

Frei C, Opitz I, Soltermann A et al (2011) Pleural mesothelioma side populations have a precursor phenotype. Carcinogenesis 32:1324 1332. doi:10.1093/carcin/bgr127
Jongsma J, van Montfort E, Vooijs M et al (2008) A conditional mouse model for malignant mesothelioma. Cancer Cell 13:261-271. doi: 10.1016/j.ccr.2008.01.030

Ke YY, Reddel RRR, Gerwin BIB et al (1989) Establishment of a human in vitro mesothelial cell model system for investigating mechanisms of asbestos-induced mesothelioma. Am J Pathol 134:979-991

Kroczynska B, Cutrone R, Bocchetta M et al (2006) Crocidolite asbestos and SV40 are cocarcinogens in human mesothelial cells and in causing mesothelioma in hamsters. Proc Natl Acad Sci U S A 103:14128-14133. doi:10.1073/pnas. 0604544103

La Rocca PJ, Rheinwald JG (1985) Anchorage-independent growth of normal human mesothelial cells: a sensitive bioassay for EGF which discloses the absence of this factor in fetal calf serum. In Vitro Cell Dev Biol 21:67-72

Levresse V, Renier A, Levy F et al (2000) DNA breakage in asbestostreated normal and transformed (TSV40) rat pleural mesothelial cells. Mutagenesis 15:239-244

Pietruska JR, Kane AB (2007) SV40 oncoproteins enhance asbestosinduced DNA double-strand breaks and abrogate senescence in murine mesothelial cells. Cancer Res 67:3637-3645. doi:10.1158/ 0008-5472.CAN-05-3727

Provost JJ, Rastedt D, Canine J et al (2012) Urokinase plasminogen activator receptor induced non-small cell lung cancer invasion and metastasis requires NHE1 transporter expression and transport activity. Cell Oncol 35:95-110

Raschke WC, Baird S, Ralph P, Nakoinz I (1978) Functional macrophage cell lines transformed by Abelson leukemia virus. Cell 15:261-267

Rascoe PA, Jupiter D, Cao X et al (2012) Molecular pathogenesis of malignant mesothelioma. Expert Rev Mol Med 14:e12. doi:10. 1017/erm.2012.6

Robinson C, van Bruggen I, Segal A et al (2006) A novel SV40 TAg transgenic model of asbestos-induced mesothelioma: malignant transformation is dose dependent. Cancer Res 66:10786-10794. doi:10.1158/0008-5472.CAN-05-4668

Sekido YY, Pass HIH, Bader SS et al (1995) Neurofibromatosis type 2 (NF2) gene is somatically mutated in mesothelioma but not in lung cancer. Cancer Res 55:1227-1231

Sherwood AL, Mutsaers SE, Peeva VK et al (2008) Spontaneously immortalized mouse mesothelial cells display characteristics of malignant transformation. Cell Prolif 41:894-908. doi:10.1111/j.13652184.2008.00560.x

Smalley KSMK, Lioni MM, Herlyn MM (2006) Life isn't flat: taking cancer biology to the next dimension. In Vitro Cell Dev Biol Anim 42:242-247. doi:10.1290/0604027.1

Zucali PA, Ceresoli GL, De Vincenzo F et al (2011) Advances in the biology of malignant pleural mesothelioma. Cancer Treat Rev 37: 543-558. doi:10.1016/j.ctrv.2011.01.001

Zurita E, Chagoyen M, Cantero M et al (2011) Genetic polymorphisms among C57BL/6 mouse inbred strains. Transgenic Res 20:481-489. doi:10.1007/s11248-010-9403-8 